\title{
Gestión financiera en planes de ordenamiento territorial como herramienta de desarrollo urbano
}

\section{Financial management in land management plans as a tool for urban development}

\author{
Ronal Aníbal Zambrano - Mercado ${ }^{1}$, Helmer Muñoz - Hernández², Cristian Camilo Brito - \\ Aleman $^{3}$, Angie Patricia Caro - Flórez ${ }^{4}$ \\ ${ }^{1}$ Estudiante del programa de Contaduría Púbica, Universidad del Sinú Elyas Bechara Zainum, Colombia, \\ ORCID: https://orcid.org/0000-0002-4454-5446, E-mail: ronalzambrano@unisinu.edu.co \\ ${ }^{2}$ Docente programa Contaduría Pública, Investigador Asociado y Par Evaluador de Colciencias, \\ Universidad del Sinú Elyas Bechara Zainum Colombia, ORCID: https://orcid.org/0000-0002-2445-6585, \\ E-mail: helmermunoz@unisinu.edu.co
}

${ }^{3}$ Estudiante del programa de Contaduría Púbica de la Universidad del Sinú Elyas Bechara Zainum, Colombia, ORCID: https://orcid.org/0000-0003-0430-4768, E-mail: cristianbrito@ unisinu.edu.co

${ }^{4}$ Estudiante del programa de Contaduría Púbica de la Universidad del Sinú Elyas Bechara Zainum, Colombia, ORCID: https://orcid.org/0000-0003-1287-439X, E-mail: angiecaro@unisinu.edu.co

Cómo citar: Zambrano - Mercado, R. A., Muñoz - Hernández, H., Brito - Aleman, C. C., \& Caro - Florez, A. P. (2020). Gestión financiera en planes de ordenamiento territorial como herramienta de desarrollo urbano. Revista Científica Profundidad Construyendo Futuro, 13(13), 31-38. https://doi.org/10.22463/24221783.2577

Recibido: 03 de Mayo de 2020 / Aprobado: 15 de Junio de 2020

\begin{abstract}
Resumen
El desarrollo de este artículo con base a una metodología descriptiva, de tipo documental, bajo un enfoque cualitativo, describe la relevancia de la gestión financiera en la formulación de instrumentos de Desarrollo y Ordenamiento territorial en los entes territoriales, como instrumentos que derivan el desarrollo urbano de los mismos. Con ello, se resalta la correcta planificación, así como manejo contable por medio de sistemas contables adecuados, que posteriormente permitirán la correcta gestión financiera, en pro de planes de ordenamiento sostenibles. Con base a ello, se precisa el objetivo general de; establecer cómo la gestión financiera en los planes de ordenamiento territorial funciona como una herramienta de desarrollo urbano evaluando. Esto a partir del estudio de la viabilidad de su consecución y la asignación de recursos financieros. Esto, teniendo en cuenta que ésta, permite la construcción de instrumentos financieros que son apoyo e insumos para la preparación y realización de los Planes de Desarrollo y de Ordenamiento Territorial. Instrumentos tales como como el Plan Operativo Anual de Inversiones, el Marco Fiscal a mediano Plazo y demás planes financieros, así como la disposición de políticas públicas que impulsen esta planeación consecutiva, que permita la utilización proyectada del capital financiero utilizables en el ente territorial, para la ejecución de programas y proyectos de manera que estos que ejecuten eficientemente y generando un beneficio común.
\end{abstract}

Palabras clave: Desarrollo urbano; Ente territorial; Gestión financiera, Plan de Ordenamiento Territorial, Política contable.

\section{Abstract}

The development of this article based on a descriptive methodology, highlights the importance of financial management in the formulation of Development Plans and Territorial Planning in territorial entities, as instruments 
that derive their urban development. With this, correct planning is highlighted, as well as accounting management through adequate accounting systems, which will subsequently allow correct financial management, in favor of sustainable management plans. Based on this, the general objective of; establish how financial management is closely related to planning in territorial entities through the Development Plan and Territorial Planning, evaluating the viability of its achievement and the allocation of financial resources. This, taking into account that this one, allows the construction of financial instruments that are support and inputs for the elaboration and execution of the Development and Territorial Planning Plans. Instruments such as the Annual Investment Operational Plan, the Medium-Term Fiscal Framework and other financial plans, as well as the provision of public policies that promote this consecutive planning, which allows the projection of the financial resources available in the territorial entity for the execution of programs and projects so that they execute efficiently and generating a common benefit.

Keywords: Urban Development; territorial entity; financial management, land use plan, accounting policy.

\section{Introducción}

La gestión contable se entiende como aquella gestión que busca la elaboración de una observación e inspección de las actividades financieras que ejecute una organización. Esta, resulta indispensable para la proyección y el bosquejo de programas económicos que permitan que todas esas operaciones financieras se ejecuten de forma correcta y con ello, anticipar amenazas económicas antes de que ocurran. En este sentido, se precisa que la contabilidad permite conocer el manejo contable, financiero y económico de una organización, así como los resultados de la gestión empresarial que se realice; por medio de una organización y presentación a través de informes financieros. Por otro lado, se precisa que, de acuerdo al DNP, el Plan de Desarrollo de un ente territorial es:

El instrumento de planificación que orienta el accionar de los diferentes actores del territorio durante un período de gobierno, en él se expresan los objetivos, metas, políticas, programas y proyectos de desarrollo, así como los recursos para su financiación (Plan Plurianual de Inversiones). (DNP, 2012)

Así mismo, las administraciones deberán elaborar un Plan de Ordenamiento Territorial, que es una herramienta esencial, establecida en la Ley 388 de 1997, para que los entes territoriales del país, proyecten la organización de su territorio. En este POT se ordena el área urbana y rural a través de programas y proyectos los cuales buscan acercar al territorio a la visión de lo que se desea del municipio, del cual, a su vez, se deriva el desarrollo urbano del ente territorial.

Con base a estas precisiones, se desarrolla el presente artículo, sobre el cual se destaca una estructura que inicialmente, relaciona los conceptos de: gestión financiera y la gestión contable, a partir de los elementos preliminares que esta última aporta, en la correcta planificación de recursos propios de la gestión financiera. Posteriormente se expone el origen de la importancia de planificación del territorio y como se desenvuelve el desarrollo urbano en esta planificación y por último se establecerá la relación que existe entre la planificación del territorio y la ejecución de una gestión financiera en el ente. Finalmente, se establecen las conclusiones, resaltando que la gestión financiera, permite la construcción de instrumentos financieros que son apoyo e insumos para la elaboración y ejecución de los POT; permitiendo la preparación de los recursos aprovechables, para la práctica de estrategias y proyectos de forma eficiente $y$ eficaz. 


\section{Marco teórico}

Existen conceptos clave a partir de las teorías y enfoques del ordenamiento territorial; que, por medio de la gestión del desarrollo, permiten la comprensión y ejecución adecuada de la gestión financiera en pro del desarrollo urbano de los entes territoriales. Con base a ello, inicialmente, se destaca que la gestión urbana, se define como: "un mecanismo de gestión del suelo, con el objeto de asegurar el reparto equitativo de cargas y beneficios en el suelo urbano, con tratamientos de renovación urbana y/o redesarrollo o actualización" (Ministerio de Vivienda, ciudad y territorio, 2015), logrando una mejor distribución de espacios y uso, de la cual depende el adecuado desarrollo urbano que ostenta y logra un ente territorial. Como gestión, está de la mano con todas las decisiones gerenciales que el operador del gasto, por medio de su equipo de trabajo debe liderar, para garantizarlo. $\mathrm{Y}$ es ahí donde entran los elementos de gestión contable, financiera, planificación, control, entre otros, que, de forma dinámica, garantizarán dichos fines de desarrollo urbano.

Por otro lado, se tiene en cuenta que la gestión en contabilidad busca facilitar las metas planteadas por la organización, cuyo principal objeto es "predecir el flujo de efectivo de dinero y bienes que posee la empresa, los cuales conforman el patrimonio, colaborando así, con la toma de decisiones relacionadas con inversiones, ventas y solicitudes de créditos", (GQ Asesores, 2016) permitiendo una adecuada verificación de las actividades realizadas en una organización. Y, como parte de esta gestión contable, se encuentra la gestión financiera, la cual se define como: "la actividad que se realiza en una organización para planificar, organizar, dirigir, controlar y monitorear todo el manejo de los recursos financieros (activos con grado de liquidez) con el fin de generar mayores beneficios y/o resultados para la organización.” (Terrazas Pastor, 2009).

La meta principal en la gestión financiera para los municipios, distritos y departamentos, es mejorar la destinación de los recursos financieros logrando así un desarrollo y bienestar común, que vaya de la mano con la consolidación de satisfacción de las necesidades básicas de la población. Por esto, es imperante que, en esta gestión, se elabore un diagnóstico integral de la situación del municipio, que en muchas ocasiones se representa a través de un árbol de problemas que permita relacionar las raíces y las consecuencias del contexto financiero del ente territorial. En este sentido, algunas causas que generan pérdidas financieras o desorden administrativo son: mal manejo de recursos financieros, humano o técnicos, mala política salarial e incluso un mal clima laboral.

De igual forma, también se plantea un modelo de gestión financiera a través de un árbol de objetivos de la situación financiera estable de la entidad. En este árbol se describe un objetivo general, sus objetivos específicos y los resultados esperados. Por ejemplo, para generar garantías financieras $y$ orden administrativo, se deberá realizar un buen manejo del recurso financiero, humano y técnico, definir una correcta política salarial y mejorar el clima laboral. Este modelo de gestión financiera precisa de cuatro fases, dinamizadas en la figura 1 , de la cual se establece que:

- Planificación de actividades y planificación financiera presupuestal, donde se generan las políticas y estrategias de la organización.

- Ejecución y análisis de la información financiera, se hace teniendo como base una 
preparación presupuestaria previa $\mathrm{y}$ permitirán registrar la información financiera de la organización

- Control y apoyo a la toma de decisiones, busca monitorear las acciones realizadas y apoyar la toma de decisiones.

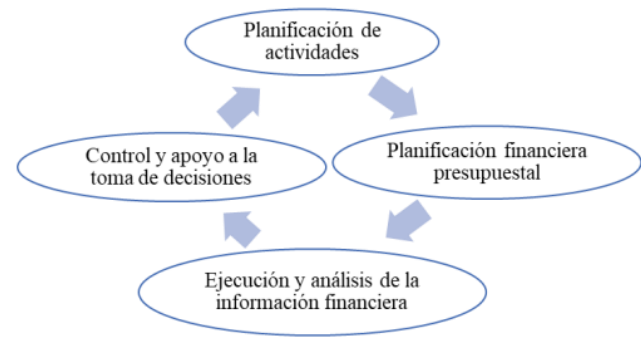

Figura 1. Modelo de gestión financiera. Fuente: creación propia.

\section{Metodología}

Este artículo, se desarrolló por medio un estudio de tipo documental, bajo un método descriptivo, con enfoque cualitativo; basado en la recopilación de información y posterior análisis de la misma. A partir de ello, desglosa la relación de los conceptos clave, de tal forma que, se aporten elementos investigativos objetivos que permitan mejorar la utilización de estos instrumentos de planificación territorial, por medio la correcta aprehensión que la gestión contable dinamiza para ello, por medio de una adecuada planificación estratégica para ello, entre otros aspectos.

\section{Resultados}

\subsection{Desarrollo integral en el ordenamiento territorial}

Teniendo como base la Guía para la Gestión Pública Territorial $\mathrm{N}^{\circ} 4$, define el desarrollo integral como un derecho fundamental, revestido de reconocimiento internacional. Con base a ello, este desarrollo se presenta como un proceso sistematizado, con varias dimensiones que propende la transformación a favor de los intereses de la población, "en armonía y equilibrio con lo ambiental, lo socio-cultural, lo económico y lo político administrativo en un territorio determinado"

Ahora bien, para entender de qué manera funciona el territorio, se debe tener en cuenta que este involucra la interrelación entre la naturaleza y los elementos poblacionales que forman parte de un mismo sistema. Y, entre esas dimensiones clave, se encuentra la dimensión económica, la cual se da lugar a las formas de adjudicación, realización, comercialización y gasto de los recursos en el territorio, que, de acuerdo al Departamento Nacional de Planeación, dispone la ordenación para asegurar los elementos para la reproducción y la "sostenibilidad de la población, del ambiente y de la organización social. Desde el punto de vista de la competitividad territorial, esta dimensión se orienta a la construcción de sistemas productivos territoriales que propicien el desarrollo económico local." (Departamento Nacional de Planeación, 2011)

Este ordenamiento del territorio se da a través del Plan de Ordenamiento Territorial, en adelante POT, que es un mecanismo para elaborar los procesos de organización de los territorios. Con base a ello, se define como el sistema de objetos, criterios, estrategias, metas, actividades y normas dispuestas "para orientar y administrar el desarrollo físico del territorio y la utilización del suelo". (Ley 388, 1997). Y es que estos instrumentos, cumplen el objetivo de perfeccionar la planeación socioeconómica a fin de "racionalizar las intervenciones sobre el territorio y orientar su desarrollo y aprovechamiento sostenible". Esta organización insta tres estrategias básicas: 
- La organización del uso de la tierra

- Proporción de los espacios con relación a los programas de inversión local y económica

- Organización funcional y administrativa óptima del territorio. (Bautista y Malagón, 2008).

De acuerdo a Jesús Bautista y Olga Malagón, "La planeación territorial o regional es la determinación de las directrices de la organización urbanística y el ordenamiento de las actividades humanadas en el territorio de una región determinada; atendiendo a las necesidades sociales y económicas, posibilidades y recursos de dicho territorio." (Bautista y Malagón, 2008).

Por su parte, se debe resaltar que el desarrollo urbano se encuentra inmerso en el ordenamiento del territorio debido a que este debe, coordinar, orientar, dirigir el seguimiento y valoración de las pautas de desarrollo urbano, vivienda y agua potable y saneamiento básico en el territorio. En este sentido, tienen la misión de "promover el fortalecimiento de la infraestructura social y el ordenamiento y mejora de la planificación de los centros urbanos del país." (DNP, s.f.).

\subsection{Inclusión de la gestión financiera en los planes de desarrollo y de ordenamiento del territorio.}

Con base al Instituto para el Desarrollo de Antioquia, "La gestión financiera en el sector público puede entenderse como el manejo de los recursos económicos disponibles por una Entidad Territorial u otra agencia de carácter público, para garantizar la provisión de bienes y servicios a la ciudadanía." (Instituto para el Desarrollo de Antioquia IDEA, 2015, pág. 4). Es decir, en los entes territoriales, la gestión financiera permitirá garantizar que los bienes y servicios se distribuyan de manera correcta la población. Esta gestión deberá ser planificada para poder cumplir con sus objetivos. Es por eso que, se elaboran planes enfocados a la gestión financiera que son insumos para elaboración del Plan de Desarrollo, como:

- Plan Operativo Anual de Inversiones, que es la herramienta que busca concretar las inversiones para los programas y metas debidamente diseñadas en el Plan de Desarrollo del ente territorial.

- Marco Fiscal de mediano plazo, que se enfoca en los objetivos y resultados y de la política fiscal del ente territorial. En este documento se computan los comportamientos de la actividad económica en un año anterior, así como estimaciones y consistencia de las cifras presupuestales para el año en curso. (Ministerio de Hacienda y Crédito Público, s.f.)

Estos instrumentos de planificación financiera del sector público territorial no son más que un programa de entradas y egresos de caja, con las respectivas alternativas de financiamiento. "A través de este instrumento es posible establecer cuáles son los recursos disponibles para la ejecución del Plan de Desarrollo y su monto." (DNP, 2012). Igualmente, como en los Planes de Desarrollo, los POT también están relacionados estrechamente con la gestión financiera del ente territorial porque esta última también permitirá mejorar la distribución de los recursos necesarios para la puesta en marcha de los proyectos establecidos en estos Planes de Ordenamiento Territorial. "En la gestión financiera es fundamental comprender y entender instrumentos de planeación como el Plan de Ordenamiento Territorial, que da pautas en términos de usos del suelo." (Instituto para el Desarrollo de Antioquia IDEA, 2015, pág. 12). 
La gestión financiera de los POT inicia con la definición del alcance y los objetivos del instrumento de misión de las finanzas. Esta etapa se realiza una vez se aprueba la movilización del capital que se necesita para la ejecución financiera. Sobre esto, el primer paso es estimar lo que puede costar la implementación del POT en un municipio.

Las proyecciones de los costos se realizan $\mathrm{y}$ se redefinen teniendo en cuenta las instrucciones de organización, que establecen la trascendencia de los tiempos de trabajo. Sobre ello, se indica que al trabajo se le da viabilidad si se acredita que los beneficios son mayores a los costos, y así mismo, "que la organización no puede obtener un mejor retorno al invertir los mismos fondos en otros proyectos (Praxis, 2020).

\subsection{Beneficios del manejo adecuado de la gestión financiera para el impulso del desarrollo urbano, por medio de los POT.}

La planeación del desarrollo urbano se adoptó en la Ley 388 de 1997 de Desarrollo Territorial, que obliga a las entidades territoriales a formular el POT y buscar la recuperación de la planeación urbana en los territorios urbanos principalmente. Inicialmente los POT buscaban adaptar la planeación del territorio a nuevas tendencias de urbanismo internacional. Sin embargo, se debió enfatizar en la gestión de financiación de este desarrollo urbano para hacer posible y viable la ejecución de estos planes. De esta necesidad, nacen algunos instrumentos de financiación permitiendo así que estos planes pudieran volverse realidad.

En Colombia, "a través de la acción coordinada de los Ministerios de Hacienda, Desarrollo y el Departamento Nacional de Planeación y dentro del marco del Programa de Fortalecimiento del Desarrollo Territorial contenido en la política para la consolidación de la descentralización, se diseñan planes y programas de apoyo técnico a las ciudades en aspectos financieros para el manejo de los asuntos urbanos." (Ciudades y Ciudadanía, 1995). Uno de estos, es el Plan de Apoyo al Fortalecimiento Financiero Urbano, el cual establece mecanismos para:

- Determinar las condiciones de apalancamiento del desarrollo urbano, en un nivel tributario y crediticio actual y a futuro.

- Identificar faltas de administración financiera que busque dar cumplimiento a los programas urbanísticos.

- Trazar líneas metodológicas en pro de la planificación y la gestión financiera en el nivel urbano, a partir de la disposición de los procesos de presupuesto y organización del territorio.

- Diseñar estrategias de verificación de los resultados relacionados con la calidad del gasto público a nivel urbano.

De esta manera, el gobierno a través de sus instituciones garantiza un desarrollo urbano a través de la planificación del territorio y recurre a la gestión financiera para articular esta planificación con los procesos presupuestales y monitorear el gasto público urbano.

\section{Conclusiones}

Sobre este artículo, se concluye que la gestión contable se dispone para contribuir en el cumplimiento de las metas planteadas por el ente territorial, en este caso la entidad territorial, del cual se resalta la finalidad de pronosticar el comportamiento del dinero en efectivo y los bienes disponibles que conforman el patrimonio de la organización.

De esta manera, se mejora el proceso relacionado con la toma de decisiones con 
relación a las inversiones, negocios comerciales, compras y ventas y manejo crediticio; dentro de la cual se encuentra la gestión financiera dirigidas a las operaciones que de la organización para planificar, organizar, dirigir, controlar y monitorear todo el manejo de los recursos financieros (activos con grado de liquidez) con el fin de generar mayores beneficios y/o resultados para la organización.

En este orden de ideas, la gestión financiera, inmersa en la gestión contable de los entes territoriales permite la construcción de instrumentos financieros que son apoyo e insumos para la preparación y realización de los Planes de Desarrollo y POT. A su vez, existen herramientas complementarias que permiten pronosticar la utilización de los recursos financieros disponibles en los entes territoriales para la ejecución de programas y proyectos de manera que estos que ejecuten eficientemente $\mathrm{y}$ generando un beneficio común. Finalmente, se destaca que la administración de las finanzas es vital para el funcionamiento de las organizaciones, en este caso lo entes territoriales, cuya gestión de cubrir todos los aspectos del control de los recursos financieros; sobre las cuales se derivan ventajas tales como:

- Preparar de los costos para la ejecución de programas y proyectos.

- Examinar el contexto integral para facilitar las posibilidades de puesta en marcha de estos programas y proyectos

- Monitorear y controlar el gasto

- Control y evaluación en todo proceso de planificación para verificar que los valores de los programas y proyectos, no superen los beneficios esperados.

\section{Referencias}

Bautista, J., \& Malagón, O. (2008). Gestión del Ordenamiento Territorial.

Bogotá: Escuela Superior de Administración Pública.

Ciudades y Ciudadanía (1995). Bogotá: Departamento Nacional de Planeación.

Departamento Nacional de Planeación. (2011). Planeación para el desarrollo integral en las entidades territoriales. Bogotá.

DNP. $\quad$ (s.f.). $\quad$ Retrieved from https://www2.dnp.gov.co/programas/ vivienda-agua-y-desarrollourbano/Paginas/vivienda-agua-ydesarrollo-urbano.aspx

DNP. (2012). Guías para la Gestión Pública Territorial. Bogotá: Departamento Nacional de Planeación.

GQ Asesores (2016, Mayo 18). Retrieved from GQ Asesores: https://grasesores.com/la-gestion-contable-

como metodo-de-controlempresarial/

Instituto para el Desarrollo de Antioquia IDEA. (2015). Gestión Financiera Para Municipios. Medellín.

Ley 388. (1997). Congreso de Colombia. Manual de Políticas Contables del Instituto de Desarrollo Urbano, Vivienda y Gestión Territorial de Chía. (2017). 
Ministerio de Hacienda y Crédito Público. (s.f.). Retrieved from http://www.irc.gov.co/webcenter/port al/IRCEs/pages_conocecolombia/inf ormacinfiscal/marcofiscaldemediano plazo

Ministerio de Vivienda, ciudad y territorio. (2015). Gestión del POT. Bogotá.

Praxis. (2020, Junio 14). Retrieved from https://www.praxisframework.org/es/ knowledge/financial-management

Terrazas Pastor, R. (2009). Modelo de gestión financiera para una organización. Cochamba, Bolivia: Universidad Católica Boliviana San Pablo. 